Original Research Paper

\title{
Convergence Results for Fixed Point Problems of Accretive Operators in Banach Spaces
}

\author{
${ }^{1}$ Chioma Lydia Ejikeme, ${ }^{2}$ Mujahid Abbas and ${ }^{1}$ Dennis Ferdinand Agbebaku \\ ${ }^{I}$ Department of Mathematics, University of Nigeria, Nsukka, Nigeria \\ ${ }^{2}$ Department of Mathematics, Government College University -54000, Lahore, Pakistan
}

Article history

Received: 10-01-2020

Revised: 11-06-2020

Accepted: 28-07-2020

Corresponding Author:

Dennis Ferdinand Agbebaku

Department of Mathematics,

University of Nigeria, Nsukka,

Nigeria

Email: dennis.agbebaku@unn.edu.ng

\section{Introduction}

Let $\Omega$ be a real Banach space. The norm on $\Omega$ is represented by $\|\cdot\|$ and $\Omega^{*}$ represents the dual space of $\Omega$. Let $\Lambda \subset \Omega \times \Omega$ be an accretive operator with whose resolvent is $J_{r}^{\Lambda}, r>0$. Denote by $F\left(J_{r}^{\Lambda}\right)$, the set of fixed points of $J_{r}^{\wedge}$. This paper deals with the iterative scheme for approximating the fixed point of the resolvent of an accretive operator. That is, it is concerned with a sequence $\omega_{n}$ generated by a given iterative scheme which converges (weakly or strongly) to $\omega^{*} \in F\left(J_{r}^{\Lambda}\right)$. One of such iterative scheme is the proximal-point algorithm defined as follows: Let $\omega_{0} \in \Omega$ :

$\omega_{n+1}=J_{r_{n}}^{\Lambda} \omega_{n}, \quad n=0,1,2,3 \cdots$

Rockafellar (1976) proved that the sequence obtained from the iterative scheme (1) converges weakly. The convergence of the iterative scheme (1) in Hilbert spaces, have has been studied by many authors, see for instance (Brézis and Lions, 1978) and the references in the paper.

Güler (1991) showed, through a counter example, that the iteration (1) does not converge strongly. This lead to the modification of the proximal point algorithm in various forms so that strong convergence can be archived, see (Takahashi, 2007; Kamimura and Takahashi, 2000a).

In another paper, Kamimura and Takahashi (2000b) considered the following Halpen's type and Mann's type iterative scheme for the resolvent of $\mathrm{m}$-accretive operators defined in a reflexive Banach space:
Let $\omega_{0} \in \Omega$ :

$$
\omega_{n+1}=\alpha_{n} \omega+\left(1-\alpha_{n}\right) J_{r_{n}}^{\Lambda}+f_{n}, \quad n=0,1,2, \cdots
$$

and:

$\omega_{n+1}=\alpha_{n} \omega_{n}+\left(1-\alpha_{n}\right) J_{r_{n}}^{\Lambda}+f_{n}, \quad n=0,1,2, \cdots$

where $\alpha_{n} \subset[0,1], r_{n}>0$ and $f_{n} \subset \Omega$.

The following result for the Mann's type iterative scheme was proved.

'Theorem 1 (Kamimura and Takahashi, 2000b)

Let $\Omega$ be a uniformly convex Banach space that satisfies the Opial's condition or with Frechet differentiable norm'. Assume that the sequences $\alpha_{n} \subset$ $[0,1] r_{n}>0$ and $f_{n} \subset \Omega$ satisfies the inequalities:

i. $\lim _{n \rightarrow \infty} \sup a_{n}<1$

ii. $\liminf _{n \rightarrow \infty} r_{n}>0$

iii. $\sum_{n=0}^{\infty}\left\|f_{n}\right\|<\infty$

Let $\omega \in \Omega$ and $\omega_{n}$ be the sequence obtained from (3). If $\Lambda^{-1}(0) \neq \varnothing$, then $\omega_{n}$ converges weakly to an element of $\Lambda^{-1}(0)$.

Many real world problems in engineering and other sciences can be formulated into an initial-value problem of the form: 


$$
\frac{d x}{d t}+\Lambda x(t)=0, \quad x(0)=x_{0}
$$

Here, $\Lambda$ is an accretive operator defined in a suitable Banach space. Most evolution equations like the heat, equation are examples of such equations. Suppose that:

$$
\frac{d x}{d t}=0,
$$

then the Equation (4) becomes:

$$
\Lambda x=0
$$

The solutions of Equation (5) are the equilibrium points of differential Equation (4). Many research works have been dedicated to constructing iterative scheme for approximating these equilibrium problems, see for example (Chen and Zhu, 2008) and (Zeidler, 1985) Recently, many researchers have inroduced interesting and important iterative schemes for obtaining the zeros of accretive maps in Banach spaces, see for instance (Chang et al., 2018; 2019). Moreover, iterative scheme for finding approximate solution to differential equations have also been introduced, (Arqub, 2017; Arqub et al., 2017; 2016).

This paper considers the following three-step algorithm defined with respct to the resolvent map of an accretive operator $\Lambda$. Given that $D$ is a nonempty, closed and convex subset of a Banach space $\Omega$. Let $\omega_{0} \in D$ :

$$
\left\{\begin{array}{l}
z_{n}=\left(1-v_{n}\right) \omega_{n}+v_{n} J_{r}^{A} \omega_{n} \\
y_{n}=\left(1-\mu_{n}\right) J_{r}^{A} \omega_{n}+\mu_{n} J_{r}^{A} z_{n} \\
\omega_{n+1}=\left(1-\eta_{n}\right) J_{r}^{A} y_{n}+\eta_{n} J_{r}^{A} z_{n},
\end{array}\right.
$$

where the real sequences $v_{n}, \mu_{n}, \eta_{n} \subset(0,1)$.

\section{Preliminaries}

This section is concern with some preliminary definitions and lemmas that are to be used in the proof of the main result of his paper.

\section{Definition 1}

'Let $D$ be a closed convex and nonempty subset of $\Omega$. We say a mapping $T: D \rightarrow D$ is nonexpansive, if for all $\omega, \psi \in D^{\prime}$ :

$$
\|T \omega-T \psi\| \leq\|\omega-\psi\|
$$

Denote by $F(T)$ the fixed point set of $T$. A point $\omega \in$ $\Omega$, is a fixed point if $T \omega=\omega$. The set $F(T)$ is closed and convex, see (Goebel and Kirk, 1990). A closed and convex subset $D$ of $\Omega$ is said to have a fixed point property, if every nonexpansive mapping $T: E \rightarrow E$ has a fixed in $E$, where $E$, is a bonded, closed and convex subset of $D$. Let $J: \Omega \rightarrow 2^{\Omega}$ be the duality map defined as:

$$
J(\omega)=\left\{y^{*} \in \Omega^{*}:\left\langle\omega, y^{*}\right\rangle=\|\omega\|^{2}=\left\|y^{*}\right\|^{2}\right\}, \omega \in \Omega .
$$

\section{Definition 2}

'Let $D$ be a nonempty, convex and closed subset of $\Omega$. A mapping $T: D \rightarrow \Omega$ is firmly nonexpansive, if for all $\omega$, $\psi \in D$ there exists a map $j \in J(T \omega-T \psi)$ such that':

$\|T \omega-T \omega\|^{2} \leq\langle\omega-\psi, j\rangle$.

A firmly nonexpansive map is also nonexpansive, (Ariza-Ruiz et al., 2014a; 2014b; Bauschke and Combettes, 2011). However, the converse fails. Indeed, the mapping $T$ $=-I$, where $I$ is the identity mapping, is nonexpansive but it is not firmly nonexpansive. A characterization of firmly nonexpansive mapping is given by the following result, (Bauschke and Combettes, 2011; Goebel and Kirk, 1990).

\section{Theorem 2}

'Let $\Omega$ be a Banach space and $D \neq \varnothing$ a subset of $\Omega$. Let $T: D \rightarrow \Omega b e$ a mapping with $J$ the duality mapping. For all $\omega, \psi \in X$ the following are equivalent':

i. T is firmly nonexpansive

ii. For each $\omega, \psi \in D$ and for all $r>0$ :

$$
\|T \omega-T \omega\| \leq\|r(\omega-\psi)+(1-r)(T \omega-T \psi)\|
$$

iii. For each $\omega, \psi \in D$ and for all $0 \leq s \leq 1$ :

$$
\|T \omega-T \psi\| \leq\|(1-s)(\omega-\psi)+s(T \omega-T \psi)\| . .
$$

If $\Omega$ is a Hilbert space $H$ with the inner product $\langle\cdot, \cdot\rangle$ then $T: D \subset H \rightarrow H$ is firmly nonexpansive if for all $\omega, \psi \in D$ :

$\|T \omega-T \omega\|^{2} \leq\langle\omega-\psi, T \omega-T \psi\rangle$.

The following characterization of firmly nonexpansive maps hold in Hilbert space:

\section{Theorem 3}

'Let $H$ be a Hilbert space and $D \neq \varnothing$ a subset of $H$. For the mapping $T: H \rightarrow H$ the following are equivalent for all $\omega, \psi \in D$, (Bauschke and Combettes, 2011; Goebel and Kirk, 1990)':

- $\mathrm{T}$ is firmly nonexpansive

- $\quad I-T$ is nonexpansive

- $2 T-I$ is nonexpansive

- $0 \leq\langle T \omega-T \psi,(I-T) \omega-(I-T) \psi\rangle$, for all $\omega, \psi \in D$

- $\quad\|T \omega-T \psi\|^{2} \leq\|\omega-\psi\|^{2}-\|(\omega-T \omega)-(\psi-T \psi)\|^{2}$, for all $\omega, \psi \in D$ 
The modulus of convexity $\delta(\varepsilon)$ of $\Omega$ is defined as:

$$
\delta(\varepsilon)=\inf \left\{1-\frac{\|\omega+\psi\|}{2}:\|\omega\| \leq 1,\|\psi\| \leq 1,\|\omega-\psi\| \geq \varepsilon\right\},
$$

for every $\varepsilon$ such that $0 \leq \varepsilon \leq 2$. A Banach space $\Omega$ is uniformly convex if $\delta(\varepsilon)>0$ for every $\varepsilon>0$. For a uniformly convex Banach space, the inequality holds:

$$
\frac{\|\omega+\psi\|}{2} \leq r\left(1-\delta\left(\frac{\varepsilon}{r}\right)\right) .
$$

For every $\omega, \psi \in D$ with:

$$
\|\omega\| \leq r,\|\psi\| \leq r,\|\omega-\psi\| \geq \varepsilon .
$$

Let $D$ be a closed and convex subset of a uniformly convex Banach space $\Omega$. For each $\omega \in \Omega$ there exists a unique element $\omega^{*} \in D$ such that:

$$
\omega^{*}=P_{D} \omega \text { and }\left\|\omega-\omega^{*}\right\|=\inf \{\|\omega-\psi\|: \psi \in D\},
$$

where, $P_{D}$ is the projection map from $\Omega$ onto $D$.

\section{Definition 3 (Opial, 1967)}

'A Banach space $\Omega$ is said satisfies the Opial's condition if for any sequence $\omega_{n}$ :

$$
\omega_{n}-\psi \Rightarrow \liminf _{n \rightarrow \infty}\left\|\omega_{n}-\psi\right\|<\liminf _{n \rightarrow \infty}\left\|\omega_{n}-z\right\|,
$$

for all $z \in \Omega$ with' $z \neq \psi$.

\section{Definition 4}

'A mapping $\Lambda$ : $\Omega \rightarrow 2^{\Omega}$ with domain $D(\Lambda)=\{\omega \in \Omega$ : $\Lambda \omega \neq \varnothing\}$ and range $R(\Lambda)=\{\psi \in \Omega: z \in \Lambda \omega, \omega \in D(\Lambda)\}$ is called accretive if and only if for each $\omega, \psi \in D(\Lambda)$, there exists $j(\omega-\psi) \in J(\omega-\psi)$ that satisfy the inequality:

$\langle\Lambda \omega-\Lambda \psi, j(\omega-\psi)\rangle \geq 0$.

The mapping $A$ is accretive if and only if for each $\omega$, $\psi \in D(\Lambda)$ :

$\|\omega-\psi\| \leq\|\omega-\psi+r(\Lambda \omega-\Lambda \psi)\|$,

for all $r>0$. A mapping is $m$-accretive if it is accretive and $R(I+r \Lambda)=\Omega$ for all $r>0$.

\section{Definition 5}

Let $\Lambda: \Omega \rightarrow 2^{\Omega}$ be a multi-valued map with domain $D(\Lambda)$ and range $R(\Lambda)$ in $\Omega$. The resolvent of $\Lambda$, of order $r>$ 0 , is the multi-valued mapping $J_{r}^{\Lambda}: \Omega \rightarrow 2^{\Omega}$ defined by:

$$
J_{r}^{\Lambda}=(I+r \Lambda)^{-1} .
$$

If the operator $\Lambda$ is accretive then its resolvent is firmly nonexpansive and single-valued. The proof is given in the following Theorem which characterizes, in Banach spaces, the resolvent mapping of an accretive operator (Bruck and Reich, 1977; Goebel and Reich, 1984).

\section{Theorem 4}

'Let $\Lambda: \Omega \rightarrow 2^{\Omega}$ be an accretive operator in a Banach space $\Omega$. Then the resolvent $J_{r}^{\wedge}$ is firmly nonexpansive and single valued, for any $r>0$. Moreover, any firmly nonexpansive mapping is the resolvent of an accretive operator for some' $r>0$.

Note that $F\left(J_{r}^{\wedge}\right)=\Lambda^{-1} 0=\{\psi \in \Omega: 0 \in \Lambda \psi\}$ for all $r>0$.

\section{Definition 6}

Let $\Lambda$ be an accretive operator. The Yosida approximation $\Lambda_{r}$ of $\Lambda$ is defines as:

$$
\Lambda_{r}=\frac{1}{r}\left(I-J_{r}^{A}\right) .
$$

The following Proposition gives the characterization of the Yosida approximation.

\section{Proposition 1 (Showalter, 2013)}

Let $\Lambda$ be an accretive operator and $r>0$. Then:

1. $\Lambda_{r}$ is Lipschitz with constant $\frac{2}{r}$ and $\Lambda_{r}$ is accretive on $R(I+r \Lambda)$

2. $\Lambda_{r} \omega \in \Lambda J_{r}^{\Lambda} \omega$ for all $\omega \in R\left(I+r J_{r}^{\Lambda}\right)$

3. $\left\|\Lambda_{r}\right\| \leq|\Lambda x|=\inf \{\|\psi\|: \psi \in \Lambda \omega\}$ for all $\omega \in D(\Lambda) \cap R(I$ $+r \Lambda)$

4. $\lim _{r \rightarrow 0^{+}} J_{r}^{\Lambda} \omega=\omega$, for all $\omega \in \overline{D(\Lambda)} \cap R(I+r \Lambda)$

\section{Definition 7}

Let $\Lambda: \Omega \rightarrow 2^{\Omega}$ be a multi-valued map. Let $D(\Lambda)$ denote the domain of $A$ and $R(\Lambda)$ the range in the dual $\Omega^{*}$ of $\Omega$. The map $\Lambda$ is said to be monotone if for each $\omega, \psi \in D(\Lambda)$ and any $u \in \Lambda(\omega), v \in \Lambda(\psi)$ the inequality holds:

$$
\langle u-v, \omega-\psi\rangle \geq 0 .
$$

'The operator $\Lambda$ is called a maximal monotone operator if it is a monotone operator which is not properly contained in any other monotone operator on' $\Omega$.

\section{Remark}

In any Hilbert space, the identity operator is the normalized duality mapping. The notions of accretive 
and monotone coincide in a Hilbert space. Similarly, the notions of m-accretive and maximal monotone also coincide; see (Barbu, 2010).

The following Theorems give characterizations of monotone operators in Hilbert spaces, (Minty, 1962; 1964).

\section{Theorem 5}

'Let $\Lambda: H \rightarrow 2^{H}$ be a monotone operator and $H \quad a$ Hilbert space. Then $\Lambda$ is maximal monotone if and only if $D\left(J_{r}^{\Lambda}\right)=H$, where $J_{r}^{\Lambda}$ is the resolvent of $\Omega$, for any $r>0$.'

\section{Theorem 6}

Let $T: H \rightarrow H$ be a firmly nonexpansive mapping and $\Lambda: H \rightarrow 2^{H}$ be maximal monotone. Then the following hold':

1. $B=T^{-1}-I$ is maximal monotone and $J_{r}^{B}=T$

2. $J_{r}^{A}$ is firmly nonexpansive and $\Lambda=\left(J_{\lambda}^{\Lambda}\right)^{-1}-I$,

where $J_{\lambda}^{B}$ and $J_{\lambda}^{\Lambda}$ are the resolvent of the operator $B$ and $\Lambda$ respectively.

It follows from Theorem 6 that the mapping $T \mapsto T^{-1}-I$ from the set of firmly nonexpansive mapping to the set of maximal monotone mapping is a bijection whose inverse is given as the mapping:

$$
\Lambda \mapsto J_{r}^{\Lambda}=(I+r \Lambda)^{-1} .
$$

\section{Main Result}

The proof of the main result in this study, which is the weak convergence of the sequence obtained from (6), is presented in this section. Before stating and proving the Theorem some useful Lemmas are presented.

Browder (1968) proved the following

\section{Lemma 1 (Browder, 1968)}

Let $\mathrm{D}$ be a closed, bounded and convex subset of a uniformly convex space E. Let $T: D \rightarrow D$ be a nonexpansive self-mapping. Suppose the sequence $\left\{\omega_{n}\right\}$ converges weakly to $z \in D$ and the sequence $\left\{\omega_{n}-T \omega_{n}\right\}$ converges strongly to 0 , then $T z=z$.

The main result of this paper is the following:

\section{Theorem 7}

Let $\Omega$ be a uniformly convex Banach space whose norm satisfies the Opial's condition. Let $\Lambda: \Omega \rightarrow 2^{\Omega}$ be an m-accretive operator and $J_{r}^{\Lambda}: \Omega D(\Lambda) \subset \Omega$ the resolvent of $\Lambda$ of order $r>0$. Assume that sequences $\eta_{n}$, $\mu_{n}, v_{n} \subset(0,1)$ satisfy:

1. $\limsup v_{n}<1$
2. $\lim _{n \rightarrow \infty} \mu_{n} \neq 0$

3. $\limsup \eta_{n}<1$

Let $\left\{\omega_{n}\right\}$ be the sequence obtained from (6). If $\Lambda^{-1} 0 \neq$ $\varnothing$, then $\left\{\omega_{n}\right\}$ converges weakly to $\omega^{*} \in \Lambda^{-1} 0=F\left(J_{r}^{\Lambda}\right)$.

\section{Proof}

Let $\omega^{*} \in F\left(J_{r}^{\Lambda}\right)=\Lambda^{-1} 0$. Then it follows from (6) that:

$$
\begin{aligned}
\left\|z_{n}-\omega^{*}\right\| & =\left\|\left(1-v_{n}\right) \omega_{n}+v_{n} J_{r}^{\Lambda} \omega_{n}-\omega^{*}\right\| \\
& =\left\|\left(1-v_{n}\right)\left(\omega_{n}-\omega^{*}\right)+v_{n}\left(\omega_{n}-\omega^{*}\right)\right\| \\
& \leq\left(1-v_{n}\right)\left\|\omega_{n}-\omega^{*}\right\|+v_{n}\left\|\omega_{n}-\omega^{*}\right\| \\
& =\left\|\omega_{n}-\omega^{*}\right\| .
\end{aligned}
$$

That is:

$\left\|z_{n}-\omega^{*}\right\|=\left\|\omega_{n}-\omega^{*}\right\|$.

Also:

$$
\begin{aligned}
& \left\|y_{n}-\omega^{*}\right\|=\left\|\left(1-\mu_{n}\right) J_{r}^{\Lambda} \omega_{n}+\mu_{n} J_{r}^{\Lambda} z_{n}-\omega^{*}\right\| \\
& =\left\|\left(1-\mu_{n}\right)\left(J_{r}^{\Lambda} \omega_{n}-\omega^{*}\right)+\mu_{n}\left(J_{r}^{\Lambda} z_{n}-\omega^{*}\right)\right\| \\
& \leq\left(1-\mu_{n}\right)\left\|J_{r}^{\Lambda} \omega_{n}-\omega^{*}\right\|+\mu_{n}\left\|J_{r}^{\Lambda} z_{n}-\omega^{*}\right\| \\
& \leq\left(1-\mu_{n}\right)\left\|\omega_{n}-\omega^{*}\right\|+\mu_{n}\left\|z_{n}-\omega^{*}\right\| .
\end{aligned}
$$

So that by (17):

$\left\|y_{n}-\omega^{*}\right\| \leq\left\|\omega_{n}-\omega^{*}\right\|$.

Hence from the inequalities (17) and (18) the following inequality follows:

$$
\begin{aligned}
& \left\|\omega_{n+1}-\omega^{*}\right\|=\left\|\left(1-\eta_{n}\right) J_{r}^{A} y_{n}+\eta_{n} J_{r}^{A} z_{n}-\omega^{*}\right\| \\
& =\left\|\left(1-\eta_{n}\right)\left(J_{r}^{A} y_{n}-\omega^{*}\right)+\eta_{n}\left(J_{r}^{A} z_{n}-\omega^{*}\right)\right\| \\
& =\left(1-\eta_{n}\right)\left\|J_{r}^{A} y_{n}-\omega^{*}\right\|+\eta_{n}\left\|J_{r}^{A} z_{n}-\omega^{*}\right\| \\
& \leq\left(1-\eta_{n}\right)\left\|y_{n}-\omega^{*}\right\|+\eta_{n}\left\|z_{n}-\omega^{*}\right\| \\
& \leq\left(1-\eta_{n}\right)\left\|\omega_{n}-\omega^{*}\right\|+\eta_{n}\left\|\omega_{n}-\omega^{*}\right\| \\
& =\left\|\omega_{n}-\omega^{*}\right\| .
\end{aligned}
$$

Now set $s_{n}=\left\|\omega_{n}-\omega^{*}\right\|$. Then $s_{n+1}=\left\|\omega_{n+1}-\omega^{*}\right\|$ so that inequality (19) leads to $s_{n+1} \leq s_{n}$ which implies that $s_{n}$ is decreasing. Moreover, $0 \leq s_{n+1} \leq s_{n} \leq s_{1}$ which implies $s_{n}$ is bounded. There exist a real number $M>0$ and $\left|s_{n}\right| \leq M$, hence:

$\left\|\omega_{n}\right\|-\left\|\omega^{*}\right\| \leq\left\|\omega_{n}-\omega^{*}\right\| \leq M$. 
Thus:

$$
\left\|\omega_{n}\right\| \leq M+\left\|\omega^{*}\right\|
$$

Therefore $\left\{\omega_{n}\right\}$ is bounded. From (17) and (18), it is easy to see that the sequences $\left\{z_{n}\right\}$ and $\left\{y_{n}\right\}$ are also bounded.

The inequality:

$$
\left\|J_{r}^{\Lambda} \omega_{n}\right\|-\left\|\omega^{*}\right\| \leq\left\|J_{r}^{\Lambda} \omega_{n}-\omega^{*}\right\| \leq\left\|\omega_{n}-\omega^{*}\right\| \leq M
$$

leads to:

$$
\left\|J_{r}^{A} \omega_{n}\right\| \leq M+\left\|\omega^{*}\right\|
$$

so that the sequence $\left\{J_{r}^{\Lambda} \omega_{n}\right\}$ is bounded.

Similarly:

$$
\begin{aligned}
& \left\|J_{r}^{\Lambda} y_{n}\right\|-\left\|\omega^{*}\right\| \leq\left\|J_{r}^{\Lambda} y_{n}-\omega^{*}\right\| \\
& \leq\left\|y_{n}-\omega^{*}\right\| \leq\left\|\omega_{n}-\omega^{*}\right\| \leq M,
\end{aligned}
$$

so that $\left\|J_{r}^{\Lambda} y_{n}\right\| \leq M+\left\|\omega^{*}\right\|$ and hence the sequence $\left\{J_{r}^{\Lambda} y_{n}\right\}$ is bounded. In the same way, it can be shown that the sequence $\left\{J_{r}^{\Lambda} z_{n}\right\}$ are also bounded.

Since $\left\|\omega_{n}-\omega^{*}\right\|$ is monotone decreasing and bounded, then $\lim _{n \rightarrow \infty}\left\|\omega_{n}-\omega^{*}\right\|$ exists. Without loss of generality one may assume that $\lim _{n \rightarrow \infty}\left\|\omega_{n}-\omega^{*}\right\| \neq 0$.

Since the mapping $\Lambda$ is accretive and $\Omega$ is uniformly convex, it follows that:

$$
\begin{aligned}
& \left\|J_{r}^{\Lambda} \omega_{n}-\omega^{*}\right\| \leq\left\|J_{r}^{\Lambda} \omega_{n}-\omega^{*}++\frac{r}{2}\left(\Lambda_{r} \omega_{n}-0\right)\right\| \\
& =\left\|J_{r}^{\Lambda} \omega_{n}-\omega^{*} J_{r}^{\Lambda} \omega_{n}-\omega^{*}+\frac{1}{2}\left(\omega_{n}-J_{r}^{\Lambda} \omega_{n}\right)\right\| \\
& =\left\|\frac{J_{r}^{\Lambda} \omega_{n}+\omega_{n}}{2}-\omega^{*}\right\|=\left\|\frac{J_{r}^{\Lambda} \omega_{n}-\omega^{*}+\omega_{n}-\omega^{*} \|}{2}\right\| \\
& \leq\left\|\omega_{n}-\omega^{*}\right\|\left\{1-\delta\left(\frac{\left\|\omega_{n}-J_{r}^{A} \omega_{n}\right\|}{\left\|\omega_{n}-\omega^{*}\right\|}\right)\right\} .
\end{aligned}
$$

Hence:

$$
\begin{aligned}
& \left\|\omega_{n}-\omega^{*}\right\| \delta\left(\frac{\left\|\omega_{n}-J_{r}^{\wedge} \omega_{n}\right\|}{\left\|\omega_{n}-\omega^{*}\right\|}\right) \\
& \leq\left\|\omega_{n}-\omega^{*}\right\|-\left\|J_{r}^{\Lambda} \omega_{n}-\omega^{*}\right\| .
\end{aligned}
$$

This leads to:

$$
\begin{aligned}
& \left(1-v_{n}\right)\left\|\omega_{n}-\omega^{*}\right\| \delta\left(\frac{\left\|\omega_{n}-J_{r}^{\Lambda} \omega_{n}\right\|}{\left\|\omega_{n}-\omega^{*}\right\|}\right) \\
& \leq\left(1-v_{n}\right)\left(\left\|\omega_{n}-\omega^{*}\right\|-\left\|J_{r}^{\Lambda} \omega_{n}-\omega^{*}\right\|\right) \\
& =\left(1-v_{n}\right)\left\|\omega_{n}-\omega^{*}\right\|-\left(1-\gamma_{n}\right)\left\|J_{r}^{\Lambda} \omega_{n}-\omega^{*}\right\| \\
& =\left\|\omega_{n}-\omega^{*}\right\|-\left(\left\|v_{n}\left(\omega_{n}-\omega^{*}\right)\right\|+\left\|\left(1-v_{n}\right)\left(J_{r}^{\Lambda} \omega_{n}-\omega^{*}\right)\right\|\right) \\
& =\left\|\omega_{n}-\omega^{*}\right\|-\left\|v_{n} \omega_{n}+\left(1-v_{n}\right) J_{r}^{\Lambda} \omega_{n}-\omega^{*}\right\| \\
& =\left\|\omega_{n}-\omega^{*}\right\|-\left\|z_{n}-\omega^{*}\right\| .
\end{aligned}
$$

That is:

$$
\begin{aligned}
& \left(1-v_{n}\right)\left\|\omega_{n}-\omega^{*}\right\| \delta\left(\frac{\left\|\omega_{n}-J_{r}^{A} \omega_{n}\right\|}{\left\|\omega_{n}-\omega^{*}\right\|}\right) \\
& \leq\left\|\omega_{n}-\omega^{*}\right\|-\left\|z_{n}-\omega^{*}\right\|
\end{aligned}
$$

But:

$$
\begin{aligned}
& \left\|y_{n}-\omega^{*}\right\| \leq\left(1-\mu_{n}\right)\left\|\omega_{n}-\omega^{*}\right\|+\mu_{n}\left\|z_{n}-\omega^{*}\right\| \\
& =\left\|\omega_{n}-\omega^{*}\right\|-\mu_{n}\left(\left\|\omega_{n}-\omega^{*}\right\|-\left\|z_{n}-\omega^{*}\right\|\right)
\end{aligned}
$$

which implies that:

$$
\mu_{n}\left(\left\|\omega_{n}-\omega^{*}\right\|-\left\|z_{n}-\omega^{*}\right\|\right) \leq\left\|\omega_{n}-\omega^{*}\right\|-\left\|y_{n}-\omega^{*}\right\|
$$

and:

$$
\begin{aligned}
& \left\|\omega_{n+1}-\omega^{*}\right\|=\left\|\left(1-\eta_{n}\right) J_{r}^{\Lambda} y_{n}+\eta_{n} J_{r}^{\Lambda} z_{n}-\omega^{*}\right\| \\
& \leq\left(1-\eta_{n}\right)\left\|y_{n}-\omega^{*}\right\|+\eta_{n}\left\|\omega_{n}-\omega^{*}\right\| \\
& =\left(1-\eta_{n}\right)\left\|y_{n}-\omega^{*}\right\|-\left(1-\eta_{n}\right)\left\|\omega_{n}-\omega^{*}\right\|-\left\|\omega_{n}-\omega^{*}\right\| .
\end{aligned}
$$

Thus:

$$
\begin{aligned}
& \left(1-\eta_{n}\right)\left(\left\|\omega_{n}-\omega^{*}\right\|-\left\|y_{n}-\omega^{*}\right\|\right) \\
& \quad \leq\left\|\omega_{n}-\omega^{*}\right\|-\left\|\omega_{n+1}-\omega^{*}\right\| .
\end{aligned}
$$

So that the inequality (23) can be expressed as:

$$
\begin{aligned}
& \left(1-v_{n}\right)\left\|\omega_{n}-\omega^{*}\right\| \delta\left(\frac{\left\|\omega_{n}-J_{r}^{A} \omega_{n}\right\|}{\left\|\omega_{n}-\omega^{*}\right\|}\right) \\
& \leq\left\|\omega_{n}-\omega^{*}\right\|-\left\|z_{n}-\omega^{*}\right\| \\
& \leq \frac{1}{\mu_{n}}\left(\left\|\omega_{n}-\omega^{*}\right\|-\left\|y_{n}-\omega^{*}\right\|\right) \\
& \leq \frac{1}{\mu_{n}} \frac{1}{1-\eta_{n}}\left(\left\|\omega_{n}-\omega^{*}\right\|-\left\|\omega_{n+1}-\omega^{*}\right\|\right),
\end{aligned}
$$


and thus:

$$
\begin{aligned}
& \mu_{n}\left(1-\alpha_{n}\right)\left(1-v_{n}\right)\left\|\omega_{n}-\omega^{*}\right\| \delta\left(\frac{\left\|\omega_{n}-J_{r}^{A} \omega_{n}\right\|}{\left\|\omega_{n}-\omega^{*}\right\|}\right) \\
& \leq\left(\left\|\omega_{n}-\omega^{*}\right\|-\left\|\omega_{n+1}-\omega^{*}\right\|\right) .
\end{aligned}
$$

The conditions $\limsup _{n \rightarrow \infty} v_{n}<1$ and $\limsup _{n \rightarrow \infty} \eta_{n}<1$ implies that $\left(1-v_{n}\right)>0$ and $\left(1-\eta_{n}\right)>0$ respectively. Moreover, the conditions $\lim _{n \rightarrow \infty} \beta_{n} \neq 0$ and $\lim _{n \rightarrow \infty}\left\|\omega_{n}-\omega^{*}\right\| \neq 0$, guarantee that the left side of inequality (24) is non-zero. So that, taking the limit of both sides of (24), it follows that:

$$
\begin{aligned}
& 0<\mu_{n}\left(1-\eta_{n}\right)\left(1-v_{n}\right)\left\|\omega_{n}-\omega^{*}\right\| \delta\left(\frac{\left\|\omega_{n}-J_{r}^{\Lambda} \omega_{n}\right\|}{\left\|\omega_{n}-\omega^{*}\right\|}\right) \\
& \leq\left(\left\|\omega_{n}-\omega^{*}\right\|-\left\|\omega_{n+1}-\omega^{*}\right\|\right) \rightarrow 0, \text { as } n \rightarrow \infty .
\end{aligned}
$$

Hence, it follows that $\left\|\omega_{n}-J_{r}^{\Lambda} \omega_{n}\right\| \rightarrow 0$ as $n \rightarrow \infty$.

Since $\left\{\omega_{n}\right\}$ is bounded, there exists a subsequence $\left\{\omega_{n_{j}}\right\}$ that converges weakly to $u$ in $\Omega$. Thus by Lemma $1 J_{r}^{\Lambda} u=u$ which implies that:

$$
u \in F\left(J_{r}^{\Lambda}\right)=\Lambda^{-1}(0) .
$$

\section{Uniqueness of the Weak Limit}

What remains is to prove that the weak limit is unique in $F\left(J_{r}^{\Lambda}\right)$. Assume that $\Omega$ satisfies the Opial's condition. Let $u_{1}, u_{2}$ be two weak sub-sequential limits of the subsequence $\left\{\omega_{n_{j}}\right\}$ such that $\left\{\omega_{n_{j}}\right\}$ converges weakly to $u_{1}$ and $u_{2}$. Then $u_{1}, u_{2} \in F\left(J_{r}^{\Lambda}\right)$.

\section{Claim: $u_{1}=u_{2}$}

The proof is by contradiction. Suppose $u_{1} \neq u_{2}$. By the Opial's condition:

$$
\begin{aligned}
& \liminf _{n \rightarrow \infty}\left\|\omega_{n}-u_{1}\right\|=\liminf _{n \rightarrow \infty}\left\|\omega_{n_{j}}-u_{1}\right\| \\
& <\liminf _{n \rightarrow \infty}\left\|\omega_{n_{j}}-u_{2}\right\|=\liminf _{n \rightarrow \infty}\left\|\omega_{n}-u_{2}\right\| \\
& =\liminf _{n \rightarrow \infty}\left\|\omega_{n_{i}}-u_{2}\right\|<\liminf _{n \rightarrow \infty}\left\|\omega_{n_{i}}-u_{1}\right\| \\
& =\liminf _{n \rightarrow \infty}\left\|\omega_{n}-u_{1}\right\|,
\end{aligned}
$$

which is a contradiction. Hence $u_{1}=u_{2}$. Thus the sequence $\left\{\omega_{n}\right\}$ converges weakly to a unique element in $F\left(J_{r}^{\Lambda}\right)=\Lambda^{-1}(0)$. This completes the proof.

\section{Applications}

Suppose $H$ is a Hilbert space, the function $f: \mathrm{H} \rightarrow \mathbb{R} \cup\{\infty\}$ is a proper lower semi-continuous and convex function. Its sub-differential, denoted by $\partial f$, is given as:

$$
\begin{aligned}
& \partial f(\omega)=\{z \in \mathrm{H}: f(\psi) \\
& \geq f(\omega)+\langle\psi-\omega, z\rangle, \text { for all } \omega, \psi \in H .
\end{aligned}
$$

If $\Lambda=\partial f$, then $\Lambda$ is a maximal monotone which is also an m-accretive operator see (Rockafellar, 1976). The resolvent for $\Lambda$ is defines as:

$$
J_{r}^{\Lambda} \omega=\underset{z \in \mathrm{H}}{\operatorname{argmin}}\left\{f(z)+\frac{1}{2 r}\|z-\omega\|^{2}\right\},
$$

for all $r>0$ and $\omega \in H$. If $u \in H$ is the solution to $\Lambda v=$ 0 . Then:

$$
u=\underset{z \in H}{\operatorname{argmin}} f(z)
$$

This leads to the following Corollary:

\section{Corollary 1}

Let $f: \mathrm{H} \rightarrow \mathbb{R} \cup\{\infty\}$ be a proper lower semi-continuous and convex function. Assume that the sequences $\eta_{n}, \mu_{n}, v_{n} \subset(0,1)$ are the same as in Theorem 7. Suppose $\omega_{0}$ $\in H$ is the sequence obtained from the iterative scheme:

$\left\{\begin{array}{l}J_{r}^{\Lambda} \omega_{n}=\underset{z \in H}{\operatorname{argmin}}\left\{f(z)+\frac{1}{2 r}\left\|z-\omega_{n}\right\|^{2}\right\} \\ z_{n}=\left(1-v_{n}\right) \omega_{n}+v_{n} J_{r}^{\Lambda} \omega_{n} \\ y_{n}=\left(1-\mu_{n}\right) J_{r}^{\Lambda} \omega_{n}+\mu_{n} J_{r}^{\Lambda} z_{n} \\ \omega_{n+1}=\left(1-\eta_{n}\right) J_{r}^{\Lambda} y_{n}+\eta_{n} J_{r}^{\Lambda} z_{n}, \quad n \in \mathbb{N} .\end{array}\right.$

If $F\left(J_{r}^{\Lambda}\right)=(\partial f)^{-1} 0 \neq \varnothing$, then $\omega_{n}$ converges weakly to $u \in F\left(J_{r}^{\Lambda}\right)$.

\section{Proof}

The proof follows directly from Theorem 7.

\section{Numerical Example}

Let $\Omega$ be the space of sequences $\left\{\omega_{n}\right\}$ that are squaresummable. That is:

$$
\Omega=l_{2}=\left\{\omega=\left\{e_{n}\right\} \subset \Omega: \sum_{n=1}^{\infty}\left|e_{n}\right|^{2}<\infty\right\} .
$$

Set $\Lambda: \Omega \rightarrow \Omega$ to be: 


$$
\Lambda e_{n}=\left(\frac{1}{n} e_{n}\right) .
$$

Then $\Lambda$ is continuous, linear and Accretive. The range $R(\Lambda)$ is a dense and proper subspace of $\Omega$ which is not closed. The resolvent of $\Lambda$ is given as:

$$
J_{A} e_{n}=\left(\frac{n}{n+1} e_{n}\right)
$$

Take $\eta_{n}=\mu_{n}=v_{n}=\frac{1}{2}$. A numerical computation of $\left\{\omega_{n}\right\}$ with the specified initial data is given to show that $\left\{\omega_{n}\right\}$ converges to $e_{n}^{*}=(0,0, \cdots)$ which is the fixed point of $J_{\Lambda}$. The codes used for numerical computation are written in MATLAB. The values of $\left\{\omega_{n}\right\}$ are shown in Table 1 and the convergence of $\left\{\omega_{n}\right\}$ for initial values $\omega_{0}=50$ and $\omega_{0}=-50$ is shown in Fig. 1 .

Table 1: Table showing $\left\{\omega_{n}\right\}$ values with given initial values

\begin{tabular}{llr} 
& $\omega_{0}=50$ & $\omega_{0}=-50$ \\
$\mathrm{n}$ & $\omega_{n}$ & -14.843750 \\
\hline 1 & 14.843750 & -4.406738 \\
2 & 4.406738 & -1.308250 \\
3 & 1.308250 & -0.388387 \\
4 & 0.388387 & -0.115302 \\
5 & 0.115302 & -0.034230 \\
6 & 0.034230 & -0.010162 \\
7 & 0.010162 & -0.003017 \\
8 & 0.003017 & -0.000896 \\
9 & 0.000896 & -0.000266 \\
10 & 0.000266 & -0.000079 \\
11 & 0.000079 & -0.000023 \\
12 & 0.000023 & -0.000007 \\
13 & 0.000007 & -0.000002 \\
14 & 0.000002 & -0.000001 \\
16 & 0.000001 & 0.000000 \\
\hline
\end{tabular}

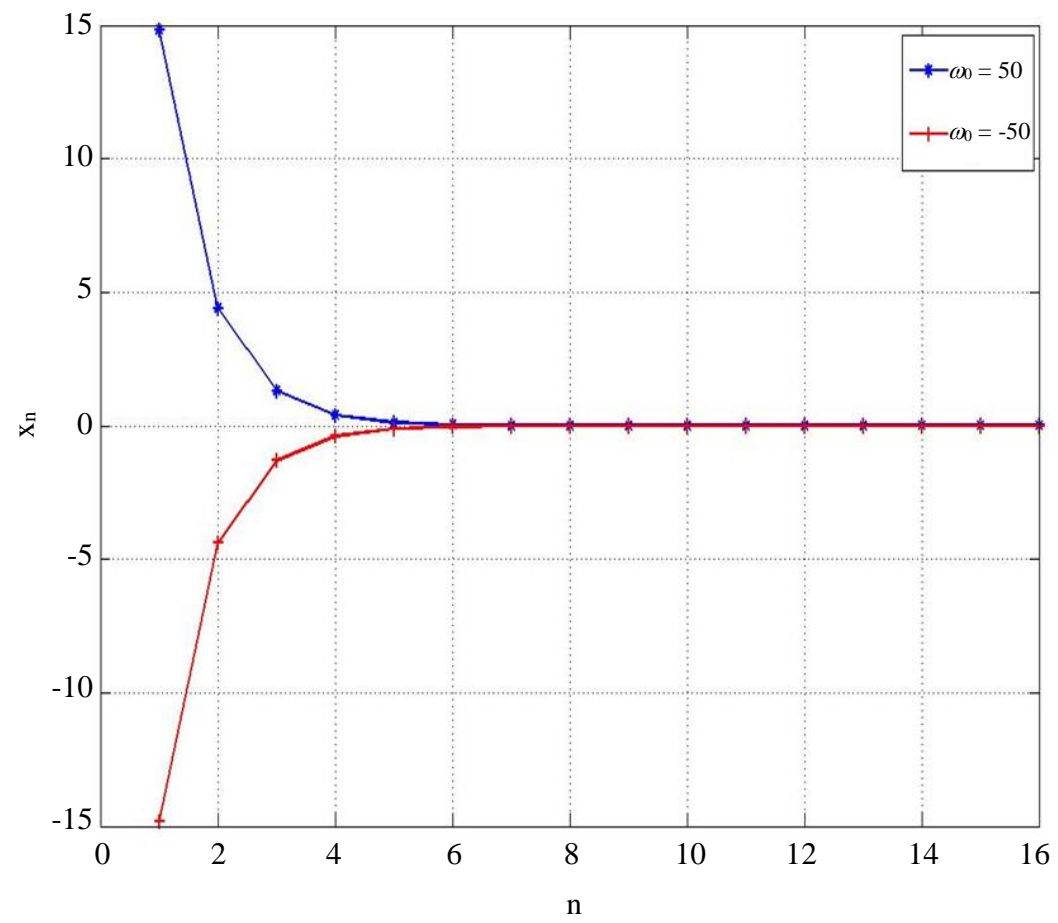

Fig. 1: Graph showing the convergence of $\omega_{n}$ 


\section{Conclusion}

A three-step iterative scheme for finding approximate solution to equations involving an accretive operator is introduced. Weak convergence of the sequence obtained from the iterative scheme was proved. A numerical example to illustrate the validity of the main result is given. From the numerical example, it is clear that the convergence of the sequence obtained from the iterative scheme is independent of the initial condition. The results obtained in this study complements some of the existing results in the literature and can be applied to some real world problem involving accretive operators.

\section{Acknowledgement}

The authors appreciates the valuable suggestions of the referees, especially for some of the references suggested by the referees.

\section{Author's Contributions}

Chioma Lydia Ejikeme: Contributed in the writing of the manuscripts.

Mujahid Abbas: Constructed the iterative scheme used in the paper. He reviewed the manuscript critically for significant intellectual content.

Dennis Ferdinand Agbebaku: Contributed in drafting, writing and in carrying out the numerical simulations.

\section{Ethics}

This article is original and contains unpublished material. The corresponding author confirms that all of the other authors have read and approved the manuscript and no ethical issues involved.

\section{References}

Ariza-Ruiz, D., Leuştean, L., \& López-Acedo, G. (2014a). Firmly nonexpansive mappings in classes of geodesic spaces. Transactions of the American Mathematical Society, 366(8), 4299-4322.

Ariza-Ruiz, D., Lopez-Acedo, G., \& Martin-Marquez, V. (2014b). Firmly nonexpansive mappings. J. Nonlinear Convex Anal, 15(1), 61-87.

Arqub, O. A. (2017). Adaptation of reproducing kernel algorithm for solving fuzzy Fredholm-Volterra integrodifferential equations. Neural Computing and Applications, 28(7), 1591-1610.

Arqub, O. A., Al-Smadi, M., Momani, S., \& Hayat, T. (2017). Application of reproducing kernel algorithm for solving second-order, two-point fuzzy boundary value problems. Soft Computing, 21(23), 7191-7206.
Arqub, O. A., Mohammed, A. S., Momani, S., \& Hayat, T. (2016). Numerical solutions of fuzzy differential equations using reproducing kernel Hilbert space method. Soft Computing, 20(8), 3283-3302.

Barbu, V. (2010). Nonlinear differential equations of monotone types in Banach spaces. Springer Science \& Business Media.

Bauschke, H. H., \& Combettes, P. L. (2011). Convex analysis and monotone operator theory in Hilbert spaces (Vol. 408). New York: Springer.

Brézis, H., \& Lions, P. L. (1978). Produits infinis de résolvantes. Israel Journal of Mathematics, 29(4), 329-345.

Browder, F. E. (1968). Semicontractive and semiaccretive nonlinear mappings in Banach spaces. Bulletin of the American Mathematical Society, 74(4), 660-665.

Bruck, R. E., \& Reich, S. (1977). Nonexpansive projections and resolvents of accretive operators in Banach spaces. Houston J. Math, 3(4), 459-470.

Chang, S. S., Wen, C. F., \& Yao, J. C. (2018). Common zero point for a finite family of inclusion problems of accretive mappings in Banach spaces. Optimization, 67(8), 1183-1196.

Chang, S. S., Wen, C. F., \& Yao, J. C. (2019). Zero point problem of accretive operators in Banach spaces. Bulletin of the Malaysian Mathematical Sciences Society, 42(1), 105-118.

Chen, R., \& Zhu, Z. (2008). Viscosity approximation method for accretive operator in Banach space. Nonlinear Analysis: Theory, Methods \& Applications, 69(4), 1356-1363.

Goebel, K., \& Kirk, W. A. (1990). Topics in metric fixed point theory (Vol. 28). Cambridge University Press.

Goebel, K., \& Simeon, R. (1984). Uniform convexity, hyperbolic geometry, and nonexpansive mappings. Dekker.

Güler, O. (1991). On the convergence of the proximal point algorithm for convex minimization. SIAM Journal on Control and Optimization, 29(2), 403-419.

Kamimura, S., \& Takahashi, W. (2000a). Approximating solutions of maximal monotone operators in Hilbert spaces. Journal of Approximation Theory, 106(2), 226-240.

Kamimura, S., \& Takahashi, W. (2000b). Weak and strong convergence of solutions to accretive operator inclusions and applications. Set-Valued Analysis, 8(4), 361-374.

Minty, G. J. (1962). Monotone (nonlinear) operators in Hilbert space. Duke Mathematical Journal, 29(3), 341-346.

Minty, G. J. (1964). On the monotonicity of the gradient of a convex function. Pacific Journal of Mathematics, 14(1), 243-247. 
Opial, Z. (1967). Weak convergence of the sequence of successive approximations for nonexpansive mappings. Bulletin of the American Mathematical Society, 73(4), 591-597.

Rockafellar, R. T. (1976). Monotone operators and the proximal point algorithm. SIAM journal on control and optimization, 14(5), 877-898.

Showalter, R. E. (2013). Monotone operators in Banach space and nonlinear partial differential equations (Vol. 49). American Mathematical Soc..
Takahashi, W. (2007). Viscosity approximation methods for resolvents of accretive operators in Banach spaces. Journal of Fixed Point Theory and Applications, 1(1), 135-147.

Zeidler, E. (1985). Nonlinear Analysis and its Applications II B: Nonlinear Monotone Operators. 